\title{
Neocortical Disynaptic Inhibition Requires Somatodendritic Integration in Interneurons
}

\author{
Court Hull, Hillel Adesnik, and Massimo Scanziani \\ Neurobiology Section, Division of Biology, University of California, San Diego, La Jolla, California 92093-0634
}

In his theory of functional polarity, Ramon y Cajal first identified the soma and dendrites as the principal recipient compartments of a neuron and the axon as its main output structure. Despite notable exceptions in other parts of the nervous system (Schoppa and Urban, 2003; Wässle, 2004; Howard et al., 2005), this route of signal propagation has been shown to underlie the functional properties of most neocortical circuits studied so far. Recent evidence, however, suggests that neocortical excitatory cells may trigger the release of the inhibitory neurotransmitter GABA by directly depolarizing the axon terminals of inhibitory interneurons, thus bypassing their somatodendritic compartments (Ren et al., 2007). By using a combination of optical and electrophysiological approaches, we find that synaptically released glutamate fails to trigger GABA release through a direct action on GABAergic terminals under physiological conditions. Rather, our evidence suggests that glutamate triggers GABA release only after somatodendritic depolarization and action potential generation at GABAergic interneurons. These data indicate that neocortical inhibition is recruited by classical somatodendritic integration rather than direct activation of interneuron axon terminals.

\section{Introduction}

The general model of information transfer in the CNS involves electrochemical communication between the axon terminal of a presynaptic cell and the somatodendritic compartments of a postsynaptic target cell. In this model, the soma and dendrites of a postsynaptic neuron integrate its excitatory and inhibitory synaptic inputs to produce an output when the membrane depolarization is sufficient to trigger an action potential. There are neuronal circuits, however, that bypass this type of somatodentritic integration. In the spinal cord, for example, glutamate release from sensory afferents is regulated by inhibitory synapses located on the axon terminal of the afferent (Rudomin, 1990). Furthermore, in the thalamus, excitatory afferent inputs target the specialized dendrites of inhibitory interneurons, which are capable of directly releasing GABA as a result of local depolarization rather than somatodendritic integration and action potential generation (Sherman, 2004).

Recent evidence from the visual cortex suggests that axons from layer $2 / 3$ pyramidal cells may directly contact the axon terminals of inhibitory interneurons, thus enabling the pyramidal cells to trigger GABA release onto their neighbors while bypassing somatodendritic integration in interneurons (Ren et al., 2007). This would allow selective and localized output from only part of the axonal arborization of an interneuron. Interneurons

\footnotetext{
Received Dec. 1, 2008; revised June 5, 2009; accepted June 8, 2009.

This work was supported by National Institutes of Health Grant NH70058. C.H. was supported by Postdoctoral National Research Service Award Fellowship 5F32NS060585-03. We are grateful to L. Petreanu and K. Svoboda for suggesting the photostimulation experiment. We thank J. Isaacson, L. Glickfeld, B. Atallah, F. Pouille, and A. MarinBurgin for their comments on this manuscript.

Correspondence should be addressed to Massimo Scanziani, Howard Hughes Medical Institute, University of California, San Diego, Neurobiology Section 0634, Division of Biology, 9500 Gilman Drive, La Jolla, CA 92093-0634. E-mail: massimo@biomail.ucsd.edu.

DOI:10.1523/JNEUROSCI.5717-08.2009

Copyright $\odot 2009$ Society for Neuroscience $\quad$ 0270-6474/09/298991-05\$15.00/0
}

would hence provide localized inhibition as specified by the anatomical pattern of pyramidal cell synapses onto their axon terminals rather than global inhibition to their cumulative target population. This type of circuit, however, contrasts with the lack of anatomical evidence for glutamatergic axoaxonic contacts in the neocortex [for discussion, see Connors and Cruikshank (2007)]. Here, we show that the observation suggesting a direct action of glutamate onto GABAergic terminals can be in part accounted for by an increased glutamate release onto the somatodendritic compartment of GABAegic interneurons using a cesium-based internal solution. Furthermore, we show that release of glutamate by direct activation of channelrhodopsinexpressing axon terminals does not drive GABAergic terminals to release GABA. We therefore find no evidence that pyramidal cells can directly activate the presynaptic terminals of neocortical interneurons to trigger GABA release under normal conditions, and conclude that neocortical inhibition is recruited by classical somatodendritic integration in these circuits.

\section{Materials and Methods}

Slice preparation. Acute coronal slices of the primary visual and somatosensory cortices $(300 \mu \mathrm{m})$ were prepared from ICR white mice [postnatal day 14 (P14) to P27] and Wistar rats (P22-P30). Mice were anesthetized with isoflurane, and rats were anesthetized with ketamine/xylazine. For experiments involving recordings from interneurons, G42 and B13 (P14-P30) mice were used (Chattopadhyaya et al., 2004; Dumitriu et al., 2007). Slices were incubated on an interface chamber for $30 \mathrm{~min}$ at $35^{\circ} \mathrm{C}$ with an artificial CSF equilibrated with $95 \% \mathrm{O}_{2}$ and $5 \% \mathrm{CO}_{2}$, containing the following (in mM): $126 \mathrm{NaCl}, 3 \mathrm{KCl}, 1.2 \mathrm{NaH}_{2} \mathrm{PO}_{4}, 1.3 \mathrm{MgSO}_{4}, 2.4$ $\mathrm{CaCl}_{2}, 26 \mathrm{NaHCO}_{3}$, and 10 glucose, $\mathrm{pH} 7.3$ and osmolarity, 310. After incubation, slices were kept in the same chamber at room temperature for $0-6 \mathrm{~h}$ until being transferred to a submerged chamber in which electrophysiological recordings were made at $31-33^{\circ} \mathrm{C}$.

Recordings. Whole-cell recordings of visually identified pyramidal cells in layer 2/3 (infrared differential interference contrast videomicroscopy and 
water-immersion $40 \times$ objective) were obtained with patch pipettes (2-5 $\mathrm{M} \Omega$ ) pulled from borosilicate capillary glass with a Sutter P-97 horizontal puller. Pipettes contained, depending on the experiment, one of the following solutions (in mM): cesium internal: 140 Cs-gluconate, 5 HEPES, $1 \mathrm{Mg}$ ATP, 10 phosphocreatine, 10 TEA (tetraethylammonium)-Cl, 1 EGTA; potassium internal: $130 \mathrm{~K}$-gluconate, $8 \mathrm{KCl}, 1 \mathrm{MgCl}_{2}, 3 \mathrm{Mg}$-ATP, $10 \mathrm{HEPES}$, 10 phosphocreatine, 1.1 or 0.2 EGTA. $\mathrm{pH}$ was adjusted to 7.2 with Cs-OH or $\mathrm{K}-\mathrm{OH}$. In a subset of repatching experiments, different Alexa dyes (488 and 594) were included in the cesium- and potassium-filled pipettes to verify that the same pyramidal cell had been patched twice. Interneurons were patched in transgenic B13 and G42 mouse lines that selectively express GFP (green fluorescent protein) in parvalbumin-expressing basket interneurons using epifluorescence.

Data acquisition and analysis. Data were recorded with a Multiclamp $700 \mathrm{~B}$ amplifier, digitized at $10-20 \mathrm{kHz}$, and analyzed off-line. In dual whole-cell recordings with a cesium internal solution, unclamped spikes ("action currents") were elicited in pyramidal cells under voltage clamp by applying a brief $(1 \mathrm{~ms})$ depolarizing voltage pulse from the holding potential of $-70 \mathrm{mV}$. With potassium internals, action potentials were generated by applying a brief $(5 \mathrm{~ms})$ depolarizing current pulse. The presynaptic cells were stimulated at $0.1 \mathrm{~Hz}$. IPSCs were recorded at the EPSC reversal potential $(0 \mathrm{mV})$ and were confirmed to be IPSCs by recording at the IPSC reversal potential $(-70 \mathrm{mV})$. Average values are expressed as mean \pm SEM. Student's $t$ test was used for statistical comparisons, with significance defined as $p<0.01$.

Latencies. The latency of postsynaptic currents was determined by the time difference between the peak of the presynaptic spike and the $5 \%$ rise point of postsynaptic currents. Despite the broad distribution of latencies between the spike evoked in a pyramidal cell and onset of IPCSs in a simultaneously recorded pyramidal cell (see Fig. $1 A$ ), even the shortest latencies are consistent with a disynaptic IPSC: The average latency of the unitary EPSC from pyramidal cells to fast-spiking (FS) cells was $0.61 \pm$ $0.04 \mathrm{~ms}(n=8)$, and the minimal latency was $0.40 \mathrm{~ms}$. The average latency of the unitary IPSC (uIPSC) from FS cells to pyramidal cells was $0.62 \pm 0.05 \mathrm{~ms}(n=8)$, and the minimal latency was $0.40 \mathrm{~ms}$. Given the fast membrane time constant and rapid kinetics of EPSPs at FS cells $[<0.5$ ms for 20-80\% rise of EPSP (Geiger et al., 1997)], and that FS cells can integrate and fire within $0.6 \mathrm{~ms}$ (Gabernet et al., 2005), a latency as short as $1.8 \mathrm{~ms}$ is a reasonable time to expect for a disynaptic connection. Based on our minimal latencies of $0.4 \mathrm{~ms}$ for monosynaptic connections, however, even shorter latencies could be expected. Indeed, in our dataset, $6 \%$ were $<2 \mathrm{~ms}$.

Estimating the fractional recruitment of interneurons. Both the study by Ren et al. (2007) and our data show average uIPSC amplitudes of $\sim 100$ $\mathrm{pA}$, and pyramidal cell evoked IPSC amplitudes of $\sim 200 \mathrm{pA}$. Given a bidirectional connection probability between fast-spiking interneurons and pyramidal cells of $0.5-0.75$ (Holmgren et al., 2003), average IPSCs result from the recruitment of four or less interneurons. To estimate what fraction of connected interneurons this represents, we evaluated the density of interneurons within a conservative cylindrical cortical volume of $200 \mu \mathrm{m}$ in diameter (even though pyramidal cells are certain to contact even more interneurons outside of this volume). Based on a cortical neuron density of $100,000 / \mathrm{mm}^{3}$, such a volume would contain 628 pyramidal cells (Holmgren et al. 2003). Although estimates of interneuron density vary (Beaulieu, 1993; Gonchar et al., 2007), if we assume $20 \%$ of layer $2 / 3$ cells are interneurons, and $50 \%$ of these interneurons are basket cells (Markram et al., 2004), then we can estimate that there are 79 basket cells in this volume. Thus, a pyramidal cell would contact $\sim 40-60$ interneurons, and only $7-10 \%$ of these (4 of $40-60)$ would need to be recruited to generate the observed IPSC.

In utero electroporation. Timed pregnant ICR white mice (Charles River) at embryonic day 15 (E15) to E16 were operated on as previously described (Saito and Nakatsuji, 2001). Each embryo was injected with1 $\mu \mathrm{g}$ of pCAGGS-ChR2-Venus (Petreanu et al., 2007) mixed with $0.5 \mu \mathrm{g}$ of pCAG-EGFP.

Channelrhodopsin-2 stimulation. Channelrhodopsin-2 (ChR2)expressing layer $2 / 3$ pyramidal cells were activated by a full-field $5 \mathrm{~ms}$ flashes of light from a $5 \mathrm{~W}$ luxeon blue light-emitting diode coupled to the epifluorescence pathway of the Olympus BX51. To record synaptic currents in the presence of TTX, we bath applied the potassium channel blocker, 4-AP, at $1 \mathrm{~mm}$ (Petreanu et al. 2009).

\section{Results}

We performed whole-cell paired recordings from neighboring $(<75 \mu \mathrm{m})$ pyramidal cells in layer $2 / 3$ of the rat somatosensory cortex. Consistent with previous findings by Kapfer et al. (2007) (0 of 305 connections) and Silberberg and Markram (2007) (4 of 1450 pairs), single action potentials triggered in one of the pyramidal cells failed to evoke disynaptic IPSCs in the neighboring pyramidal cell ( 0 of 19). To test whether these results were specific to the somatosensory cortex, we repeated these experiments in the rat visual cortex. As in the somatosensory cortex, we could not evoke disynaptic IPSCs in one of the pyramidal cells in response to individual spikes triggered in the other ( 0 of 6$)$. These results differ, however, from a recent study in the visual cortex of mice, which showed large IPSCs in layer $2 / 3$ pyramidal cells evoked by triggering a single action potential in a neighboring pyramidal cell (Ren et al., 2007).

To test whether this difference is species specific between the cortex of rats and mice, we repeated these experiments in the mouse visual cortex. As in the rat, we could not evoke disynaptic IPSCs in layer $2 / 3$ pyramidal cells in response to single spikes triggered in neighboring pyramidal cells of the mouse visual cortex (0 of 48). We did, however, observe monosynaptic EPSCs in $17 \%$ of recordings ( 8 of 48 ), consistent with the known connectivity between layer $2 / 3$ pyramidal cells [connection probability of 0.1-0.2 (Mason et al., 1991; Thomson and Deuchars, 1997; Atzori et al., 2001; Holmgren et al., 2003; Yoshimura et al., 2005; Feldmeyer et al., 2006; Kapfer et al., 2007)]. As in the visual cortex, we were also unable to evoke disynaptic IPSCs in the mouse somatosensory cortex using the same experimental protocol ( 0 of $6)$. Hence, in both the visual and somatosensory cortices of rats and mice, single action potentials evoked in layer $2 / 3$ pyramidal cells did not evoke disynaptic IPSCs in neighboring pyramidal cells ( 0 of 79 total) as previously reported.

In the study by Ren et al. (2007), disynaptic IPSCs were recruited by triggering action currents in pyramidal cells filled with a cesium-based internal solution (although a few IPSCs, 4 of 28 pairs, were generated using a potassium-based internal). Thus, to test whether this experimental difference could account for the discrepancy between our results, we performed paired recordings between pyramidal cells filled with a cesium-based internal solution (Fig. 1). Under these conditions, a brief depolarizing voltage step ( $40 \mathrm{mV}$ for $1 \mathrm{~ms}$ ) applied to a pyramidal cell held at $-70 \mathrm{mV}$ in voltage clamp produced an unclamped spike (or "action current") (Fig. 1A). In 7 of 22 of these recordings (32\%; 5 of 17 in mouse visual cortex and 2 of 5 in rat somatosensory cortex; the same percentage as observed by Ren et al.), the action current elicited large IPSCs in simultaneously recorded neighboring pyramidal cells (Fig. $1 A$ ).

To ensure that the difference between the two series of experiments was indeed attributable to different internal solutions rather than to a sampling artifact, we performed a series of paired recordings in which one of the two pyramidal cells was first patched with a potassium-based internal solution, and subsequently repatched with a cesium-based solution (Fig. $1 B$ ). Although action potentials triggered in pyramidal cells dialyzed with a potassium-based solution never evoked disynaptic IPSCs in neighboring pyramidal cells, repatching the same pyramidal cell with a cesium-based internal produced disynaptic IPSCs in 9 of 47 experiments (19\%) in the mouse somatosensory cortex, rat visual cortex, and rat somatosensory cortex (for all IPSCs re- 

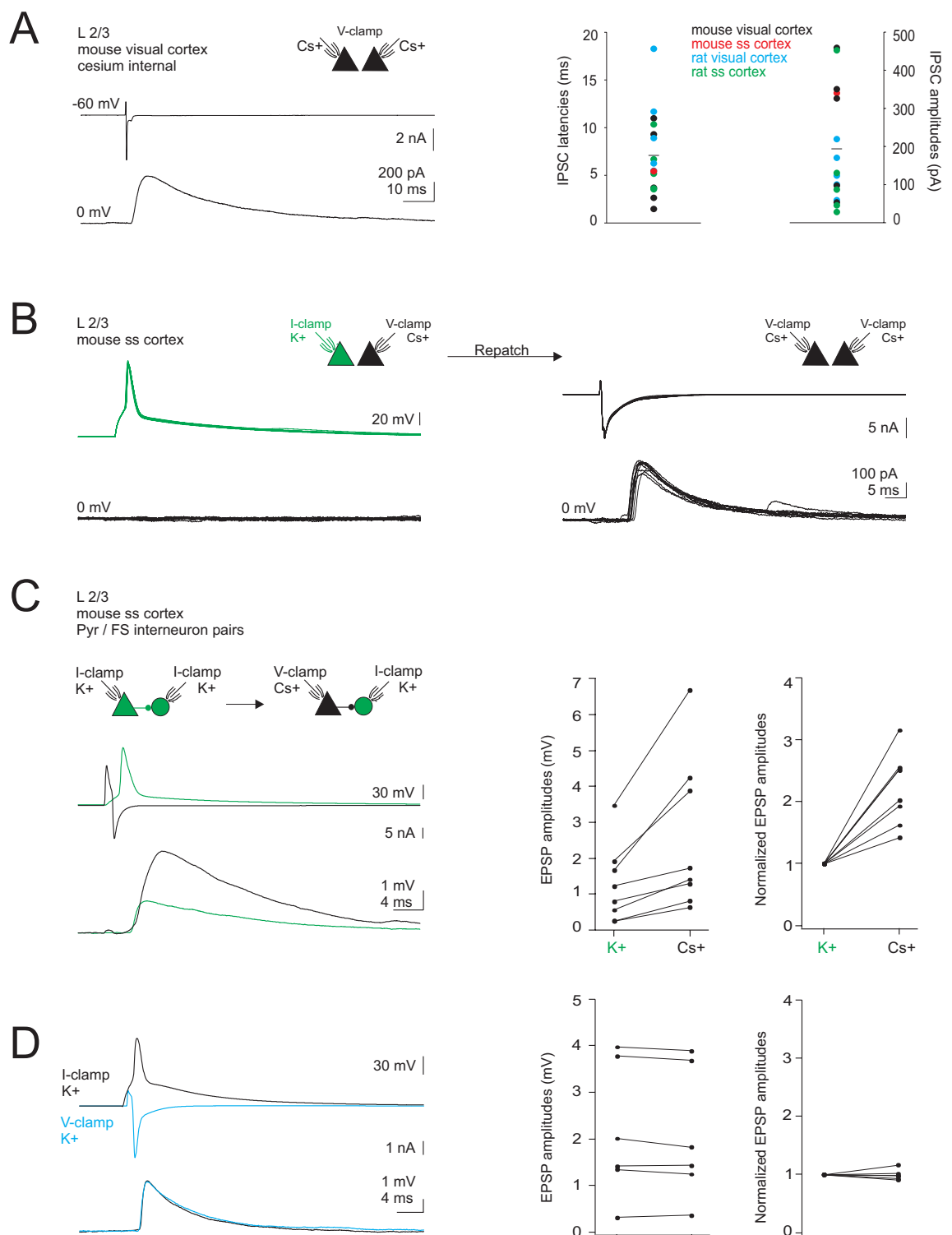

Figure 1. Increasing glutamate release from individual pyramidal cells with cesium dialysis promotes disynaptic inhibition. $\boldsymbol{A}_{\text {, }}$ IPSCS can be generated between pyramidal cell pairs in L2/3 with a cesium-based internal solution. Left, A paired recording from two nearby L2/3 pyramidal cells with a cesium internal solution in both cells. A brief depolarizing voltage step ( $40 \mathrm{mV}$ for $1 \mathrm{~ms}$ ) applied to one pyramidal cell (top) yields an unclamped spike (or "action current"). This spike produces a large IPSC in the other pyramidal cell (bottom; $0 \mathrm{mV}$ ). Right, Summary data of the latencies and amplitudes of IPSCs generated with cesium internal solutions. IPSCS were observed in 16 of $69(23 \%)$ total recordings with a cesium internal. $\boldsymbol{B}$, Repatching with a cesium-based internal solution generates IPSCS between pyramidal cell pairs. Left, A pair of pyramidal cells, in which one cell contains a potassium internal (top) and the other contains a cesium internal (bottom; $0 \mathrm{mV}$ ). Action potentials triggered in the pyramidal cell filled with potassium do not produce IPSCs in the pyramidal cell filled with cesium. Right, When the same pyramidal cell is repatched with a cesium-based internal solution, action currents (as in $\boldsymbol{A}$ ) now produce IPSCs in the other pyramidal cell. $\boldsymbol{C}$, Repatching pyramidal cells with a cesium-based internal solution doubles the size of EPSPs onto FS interneurons. Left, Paired recordings performed between a layer 2/3 pyramidal cell and an FS interneuron. The pyramidal cell is first patched with a potassium internal solution (green) and subsequently repatched with a cesium internal solution (black, as in $\boldsymbol{A}$ ). Right, Summary data. Switching from a potassium- to a cesium-based internal solution produced a 2.2-fold increase in the EPSPs recorded in FS interneurons $(n=8)$. D, The increase in FS interneuron EPSPs does not result from the voltage-clamp paradigm per se. Left, EPSP generated in an FS interneuron by triggering a spike (black) or an action current (blue) in the presynaptic pyramidal cell recorded in the current clamp or voltage clamp configuration, respectively. Right, Summary data. Note the lack of difference in the amplitude of the EPSP evoked with either recording configuration. Ss, Somatosensory.

corded in cesium: mean amplitude, $190.2 \pm 36.9 \mathrm{pA}$; mean latency, $7.1 \pm 1.1 \mathrm{~ms} ; n=16)$ (Fig. $1 B$ ). Replacing potassium with cesium did not, however, produce EPSCs between pyramidal pairs that were not already monosynaptically coupled. These re- sults suggest that a cesium-based internal solution is required to facilitate the recruitment of inhibition in response to single spikes in layer $2 / 3$ pyramidal cells.

To determine the mechanism that facilitates the recruitment of inhibition with a cesium-based internal solution, we performed paired recordings between layer $2 / 3$ pyramidal cells and FS inhibitory interneurons to compare the amplitude of monosynaptic unitary EPSPs when the presynaptic pyramidal cell was dialyzed with a potassium or cesium solution (Fig. $1 C)$. Specifically, pyramidal cells were first patched with a potassium-based internal solution, and then repatched with a cesium solution while maintaining the same postsynaptic FS interneuron. Switching from a potassium- to a cesium-based internal solution doubled the size of the unitary EPSPs recorded at FS interneurons (2.2-fold; $n=8$ ) (Fig. 1C). Furthermore, this doubling of EPSP size was directly attributable to the cesium internal, and not the voltage-clamp paradigm used to produce action currents. With a potassium internal solution, there was no difference in the amplitude of the unitary EPSP when spikes were generated in pyramidal cells with either current clamp or escape from voltage clamp ( $I$ clamp, $2.1 \pm 0.6 \mathrm{mV} ; V$ clamp, $2.1 \pm 0.6 \mathrm{mV} ; n=6$ ) (Fig. $1 D$ ).

The increased unitary excitation of inhibitory interneurons provides a plausible mechanism for the observed disynaptic IPSPs in response to a single spike in a cesium dialyzed pyramidal cell, as it has been shown previously that doubling the unitary EPSP amplitude onto interneurons strongly increases their spiking probability (Kapfer et al., 2007). However, a cesiummediated increase in synaptic transmission does not exclude the possibility suggested by Ren et al. (2007) that glutamate release may also directly activate presynaptic receptors located on GABAergic terminals and, as a consequence, trigger GABA release.

To directly address this possibility, we functionally isolated GABAergic axon terminals from the somatodendritic compartment by blocking action potentials with TTX. To trigger glutamate release from pyramidal cells in the presence of TTX, we transfected layer $2 / 3$ pyramidal cells with ChR2 through in utero electroporation of pregnant mice. Importantly, this procedure does not transfect interneurons, and therefore selectively targets pyramidal cells. The expression of $\mathrm{ChR} 2$ along the axon of pyramidal cells allows glutamate release to be triggered through photostimulation even in the absence of action potentials (Petreanu et al. 2009). 
A

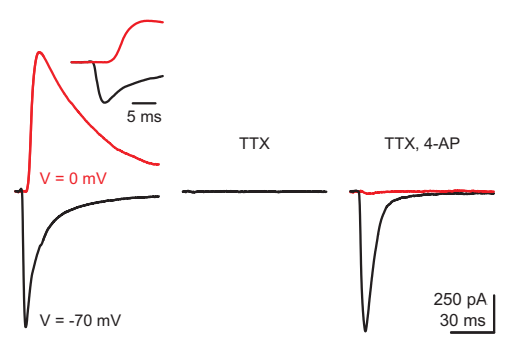

B

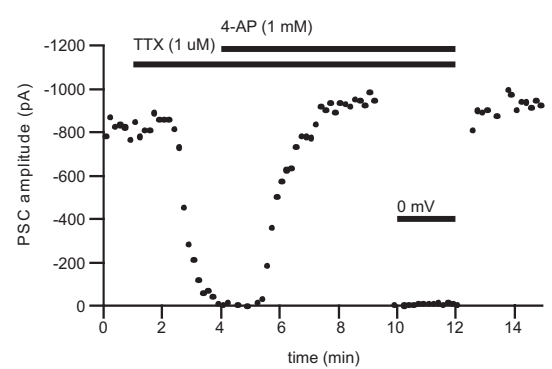

\section{C}
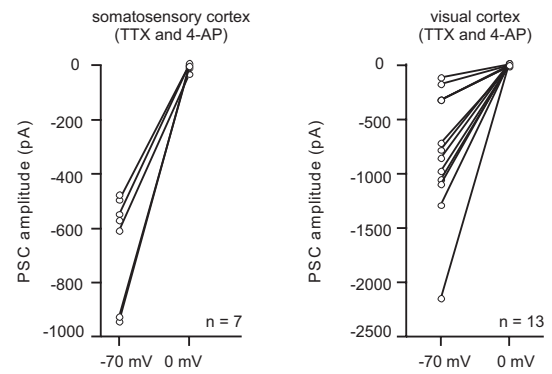

Figure 2. Strong glutamate release from pyramidal cells does not drive action potentialindependent disynaptic inhibition. $\boldsymbol{A}$, Brief flashes of blue light to ChR2-expressing L2/3 pyramidal cells drives neurotransmitter release onto neighboring ChR2-negative L2/3 cells. Cells exhibit both glutamatergic $\left(V_{\mathrm{h}}=-70 \mathrm{mV}\right)$ and disynaptic GABAergic $\left(V_{\mathrm{h}}=0 \mathrm{mV}\right)$ responses (note temporal delay in inset) in the absence of TTX (left), but only direct glutamatergic responses when action potentials are blocked and synaptic release is facilitated by the potassium channel blocker 4-AP. B, Example experiment showing the amplitude of the postsynaptic current before and during TTX application, and in the additional presence of 4-AP. The cell is held at $-70 \mathrm{mV}$ except as indicated. C, Left, Amplitude of postsynaptic currents recorded at -70 and $0 \mathrm{mV}$ in the presence of TTX and 4-AP in layer $2 / 3$ of somatosensory cortex (mean at $-70 \mathrm{mV}$, $-653 \pm 75 \mathrm{pA}$; at $0 \mathrm{mV},-5 \pm 5 \mathrm{pA} ; n=7)$. Right, Currents for cells recorded in visual cortex (mean at $-70 \mathrm{mV},-780 \pm 156 \mathrm{pA} ;$ at $0 \mathrm{mV}, 4 \pm 3 \mathrm{pA} ; n=13$ ).

Consistent with Petreanu et al. (2009), photostimulation of layer $2 / 3$ pyramidal cell axons in somatosensory cortex in the presence of TTX and 4-AP [to prolong synaptic depolarization (Kole et al., 2007; Shu et al., 2007; Goldberg et al., 2008)] could robustly evoke synaptic glutamate release, as shown by the large excitatory postsynaptic conductances recorded in pyramidal cells voltage clamped at $-80 \mathrm{mV}(8 \pm 1 \mathrm{nS} ; n=7)$ (Fig. 2). Because the unitary EPSC conductance between two layer $2 / 3$ pyramidal cells averages $0.24 \mathrm{nS}$ (Kapfer et al., 2007), photostimulation triggered the release from $\sim 30$ axons converging on the recorded pyramidal cell. To determine whether glutamate released from these photostimulated axons triggered the disynaptic release of GABA from inhibitory terminals, we voltage clamped the recorded pyramidal cell at the reversal potential of EPSCs, around $0 \mathrm{mV}$ to isolate IPSCs. Photostimulation never evoked an IPSC ( 0 of 20) as illustrated by the complete absence of an outward current in pyramidal cells (Fig. 2). We did not detect an IPSC under these conditions for stimulation of layer $2 / 3$ of somatosensory cortex $(n=7)$ (Fig. 2) or visual cortex $(n=13)$.
Hence, under the present conditions, glutamate release from pyramidal cells does not trigger GABA release by directly acting on GABAergic terminals. Rather, our data support the idea that for layer 2/3 inhibitory circuitry GABA release occurs in response to action potentials generated by classical somatodendritc integration of EPSPs.

\section{Discussion}

In agreement with the study by Ren et al. (2007), the present data show that, when layer $2 / 3$ pyramidal cells of primary sensory cortices are recorded with a cesium-containing intracellular solution, a single action current in a pyramidal cell is sufficient to trigger IPSCs at a latency consistent with disynaptic recruitment of inhibition (see Materials and Methods) in 30\% of neighboring pyramidal cells. We show that this apparent contradiction with previous observations of ours and other laboratories [i.e., in which a single spike in a pyramidal cell never or in extremely rare cases triggers inhibition in its neighbors (Mason et al., 1991; Deuchars et al., 1994; Kapfer et al., 2007; Silberberg and Markram, 2007; Lefort et al., 2009)] is likely to be attributable to the presence of cesium rather than potassium in the presynaptic recording pipette. Cesium, a potassium channel impermeant ion, can increase the probability of synaptic vesicle release by dramatically prolonging the depolarization at a presynaptic terminal during an action potential (Kole et al., 2007). Indeed, we show that intracellular cesium more than doubles the size of all unitary EPSPs, regardless of size, evoked by a pyramidal cell onto inhibitory interneurons.

Because of the high connectivity between interneurons and pyramidal cells (Holmgren et al., 2003; Kapfer et al., 2007), even the spiking of very few (one to two) interneurons in the entire slice has a high probability of being detected through an IPSC in a randomly patched pyramidal cell. The doubling of EPSP amplitudes should recruit these few interneurons in a conventional layer 2/3 disynaptic circuit. Although our data provide only a small sample of the distribution of EPSP sizes from pyramidal cells onto interneurons, previous work has shown that a significant fraction $(>10 \%)$ of these inputs are between 5 and $12 \mathrm{mV}$ (Holmgren et al., 2003). Doubling the size of these large EPSPs to $10-24 \mathrm{mV}$ would almost certainly bring some fraction of interneurons above threshold. The pyramidal cell evoked IPSCs we describe, as well as those from Ren et al., are approximately twice the amplitude of uIPSCs. Given a connection probability between FS cells and pyramidal cells of $0.5-0.75$, this suggests that IPSCs result from the recruitment of at most only four inhibitory interneurons, which corresponds to only $\sim 7-10 \%$ of the basket cells contacted by any single pyramidal cell within a cylindrical volume of cortex $200 \mu \mathrm{m}$ in diameter (Beaulieu, 1993; Holmgren et al., 2003; Markram et al., 2004; Gonchar et al., 2007) (see Materials and Methods). Unfortunately, this sparse activation of interneurons also means that direct identification of the few spiking cells will require finding the proverbial needle in a very large haystack of cortical neurons.

Contrary to the expectation that large EPSPs will spike some interneurons, Ren et al. (2007) make the provocative suggestion that at least part of the observed disynaptic inhibition of pyramidal cells is attributable to the direct action of released glutamate onto GABAergic terminals, rather than on the somatodendritic compartment of inhibitory interneurons. Furthermore, this mode of evoked GABA release does not appear to involve the recruitment of action potentials in the inhibitory axon. If true, this would allow for individual pyramidal cells to trigger disynaptic inhibition in a highly localized manner, defined by the ar- 
borization of their own axon rather than by the arborization of the recruited interneurons. To test this possibility, we used TTX to functionally uncouple the axon and somatodendritic compartment of GABAergic interneurons while evoking glutamate release via photostimulation of pyramidal cells expressing channelrhodopsin-2. Photostimulation triggered very large excitatory currents because of the release of glutamate from many axons. However, photoreleased glutamate failed to trigger GABA release, as shown by the complete absence of inhibitory currents in the recorded pyramidal cells.

Our data are thus inconsistent with a circuit involving GABA release via direct activation of glutamate receptors on presynaptic terminals of GABAergic interneurons by synaptically released glutamate. We do not, however, exclude the possibility that exogenous application of glutamatergic agonists may lead to the activation of GABAergic terminals, as shown by Ren et al. (2007). Although we believe that the presence of presynaptic cesium can explain most of the phenomena reported by Ren et al., unfortunately there are a number of inconsistencies that we are unable to replicate, including the recruitment of IPSCs with a potassium internal, the rare IPSCs with near-millisecond latencies, and the recruitment of inhibition in TTX. Our data support the hypothesis that, at least in layer $2 / 3$ of the cortex, disynaptic inhibition results from the classical integrate-and-fire behavior of inhibitory interneurons.

\section{References}

Atzori M, Lei S, Evans DI, Kanold PO, Phillips-Tansey E, McIntyre O, McBain CJ (2001) Differential synaptic processing separates stationary from transient inputs to the auditory cortex. Nat Neurosci 4:1230-1237.

Beaulieu C (1993) Numerical data on neocortical neurons in adult rat, with special reference to the GABA population. Brain Res 609:284-292.

Chattopadhyaya B, Di Cristo G, Higashiyama H, Knott GW, Kuhlman SJ, Welker E, Huang ZJ (2004) Experience and activity-dependent maturation of perisomatic GABAergic innervation in primary visual cortex during a postnatal critical period. J Neurosci 24:9598-9611.

Connors BW, Cruikshank SJ (2007) Bypassing interneurons: inhibition in neocortex. Nat Neurosci 10:808-810.

Deuchars J, West DC, Thomson AM (1994) Relationships between morphology and physiology of pyramid-pyramid single axon connections in rat neocortex in vitro. J Physiol 478:423-435.

Dumitriu D, Cossart R, Huang J, Yuste R (2007) Correlation between axonal morphologies and synaptic input kinetics of interneurons from mouse visual cortex. Cereb Cortex 17:81-91.

Feldmeyer D, Lübke J, Sakmann B (2006) Efficacy and connectivity of intracolumnar pairs of layer $2 / 3$ pyramidal cells in the barrel cortex of juvenile rats. J Physiol 575:583-602.

Gabernet L, Jadhav SP, Feldman DE, Carandini M, Scanziani M (2005) Somatosensory integration controlled by dynamic thalamocortical feedforward inhibition. Neuron 48:315-327.

Geiger JR, Lübke J, Roth A, Frotscher M, Jonas P (1997) Submillisecond
AMPA receptor-mediated signaling at a principal neuron-interneuron synapse. Neuron 18:1009-1023.

Goldberg EM, Clark BD, Zagha E, Nahmani M, Erisir A, Rudy B (2008) K+ channels at the axon initial segment dampen near-threshold excitability of neocortical fast-spiking GABAergic interneurons. Neuron 58:387-400.

Gonchar Y, Wang Q, Burkhalter A (2007) Multiple distinct subtypes of GABAergic neurons in mouse visual cortex identified by triple immunostaining. Front Neuroanat 1:3.

Holmgren C, Harkany T, Svennenfors B, Zilberter Y (2003) Pyramidal cell communication within local networks in layer $2 / 3$ of rat neocortex. J Physiol 551:139-153.

Howard A, Tamas G, Soltesz I (2005) Lighting the chandelier: new vistas for axo-axonic cells. Trends Neurosci 28:310-316.

Kapfer C, Glickfeld LL, Atallah BV, Scanziani M (2007) Supralinear increase of recurrent inhibition during sparse activity in the somatosensory cortex. Nat Neurosci 10:743-753.

Kole MH, Letzkus JJ, Stuart GJ (2007) Axon initial segment Kvl channels control axonal action potential waveform and synaptic efficacy. Neuron 55:633-647.

Lefort S, Tomm C, Floyd Sarria JC, Petersen CC (2009) The excitatory neuronal network of the $\mathrm{C} 2$ barrel column in mouse primary somatosensory cortex. Neuron 61:301-316.

Markram H, Toledo-Rodriguez M, Wang Y, Gupta A, Silberberg G, Wu C (2004) Interneurons of the neocortical inhibitory system. Nat Rev Neurosci 5:793-807.

Mason A, Nicoll A, Stratford K (1991) Synaptic transmission between individual pyramidal neurons of the rat visual cortex in vitro. J Neurosci 11:72-84.

Petreanu L, Huber D, Sobczyk A, Svoboda K (2007) Channelrhodopsin-2assisted circuit mapping of long-range callosal projections. Nat Neurosci 10:663-668.

Petreanu L, Mao T, Sternson SM, Svoboda K (2009) The subcellular organization of neocortical excitatory connections. Nature 457:1142-1145.

Ren M, Yoshimura Y, Takada N, Horibe S, Komatsu Y (2007) Specialized inhibitory synaptic actions between nearby neocortical pyramidal neurons. Science 316:758-761.

Rudomin P (1990) Presynaptic inhibition of muscle spindle and tendon organ afferents in the mammalian spinal cord. Trends Neurosci 13:499-505.

Saito T, Nakatsuji N (2001) Efficient gene transfer into the embryonic mouse brain using in vivo electroporation. Dev Biol 240:237-246.

Schoppa NE, Urban NN (2003) Dendritic processing within olfactory bulb circuits. Trends Neurosci 26:501-506.

Sherman SM (2004) Interneurons and triadic circuitry of the thalamus. Trends Neurosci 27:670-675.

Shu Y, Yu Y, Yang J, McCormick DA (2007) Selective control of cortical axonal spikes by a slowly inactivating $\mathrm{K}^{+}$current. Proc Natl Acad Sci U S A 104:11453-11458.

Silberberg G, Markram H (2007) Disynaptic inhibition between neocortical pyramidal cells mediated by Martinotti cells. Neuron 53:735-746.

Thomson AM, Deuchars J (1997) Synaptic interactions in neocortical local circuits: dual intracellular recordings in vitro. Cereb Cortex 7:510-522.

Wässle H (2004) Parallel processing in the mammalian retina. Nat Rev Neurosci 5:747-757.

Yoshimura Y, Dantzker JL, Callaway EM (2005) Excitatory cortical neurons form fine-scale functional networks. Nature 433:868-873. 